\title{
Molecular characterization and reclassification of a 1.18Mbp $D M D$ duplication following positive carrier screening for Duchenne/Becker Muscular Dystrophy
}

Cinthya Zepeda-Mendoza ${ }^{1}$, Jordan Bontrager ${ }^{2}$, Camille Fisher ${ }^{3}$, Amber McDonald ${ }^{2}$, Jaya George-Abraham ${ }^{4}$, and LINDA HASADSRI ${ }^{2}$

${ }^{1}$ ARUP Laboratories

${ }^{2}$ Mayo Clinic

${ }^{3}$ Texas Oncology

${ }^{4}$ Dell Children's Medical Center of Central Texas

November 10, 2020

\begin{abstract}
A 2-month-old male harboring a duplication of DMD exons 1-7 classified as pathogenic by an outside institution presented with mildly elevated CK levels; molecular breakpoint analysis by our laboratory reclassified the duplication as likely benign. To date, proband continues to develop normally with decreased CK levels, further supporting our reclassification.

Molecular characterization and reclassification of a $1.18 \mathrm{Mbp} D M D$ duplication following positive carrier screening for Duchenne/Becker Muscular Dystrophy

Cinthya J Zepeda-Mendoza ${ }^{1}$, Jordan E Bontrager ${ }^{2}$, Camille F Fisher ${ }^{3}$, Amber McDonald ${ }^{2}$, Jaya K GeorgeAbraham $^{3}$, Linda Hasadsri ${ }^{2}$

${ }^{1}$ Cytogenetics and Genomic Microarray Laboratory, ARUP Laboratories, Salt Lake City, UT. ${ }^{2}$ Department of Laboratory Medicine and Pathology, Division of Laboratory Genetics and Genomics, Mayo Clinic, Rochester, MN. ${ }^{3}$ Dell Children's Medical Group, Austin, TX
\end{abstract}

Corresponding Author / Reprint requests:

Linda Hasadsri, M.D., Ph.D.

Division of Laboratory Genetics and Genomics

Department of Laboratory Medicine and Pathology

$2001^{\text {st }}$ St. SW, Rochester, MN 55902

Email: Hasadsri.Linda@mayo.edu

\section{Abstract}

A 2-month-old male harboring a duplication of $D M D$ exons 1-7 classified as pathogenic by an outside institution presented with mildly elevated CK levels; molecular breakpoint analysis by our laboratory reclassified the duplication as likely benign. To date, proband continues to develop normally with decreased CK levels, further supporting our reclassification.

Key clinical message 
Molecular characterization of $D M D$ duplications at the breakpoint level is necessary to accurately interpret their effects on dystrophin activity and their roles in Duchenne and Becker muscular dystrophies.

\section{Keywords}

Duchenne muscular dystrophy, DMD, duplication, dystrophinopathy

\section{Introduction}

Duchenne [DMD, MIM: 310200] and Becker [BMD, MIM: 300376] muscular dystrophies are X-linked disorders which result in progressive proximal muscle weakness and degeneration, in conjunction with characteristic elevation of creatine phosphokinase (CK) in blood. DMD is rapidly progressive and typically presents in early childhood with motor delays and gait instability, while BMD is characterized by a later-onset phenotype of skeletal muscle weakness [1]. Both DMD and BMD are caused by mutations in DMD , a $\sim 2.2 \mathrm{Mbp}$ sized gene containing 79 exons encoding the dystrophin protein. Dystrophin maintains the structural integrity of striated muscle cells through the formation of a dystrophin-glycoprotein complex, linking the cytoskeleton to the extracellular matrix and thus providing tensile strength to muscle fibers [2].

Given its size (roughly $0.1 \%$ of the human genome), a high degree of $D M D$ allelic heterogeneity exists for DMD and BMD. Deletions are the most common pathogenic variants in $D M D$ ( $\sim 4 \% \%$ of cases), followed by nucleotide substitutions (22\%), duplications (12\%), and others (inversions, insertions, etc., $\sim 2 \%$ ) [3]. These alterations disrupt dystrophin's reading frame in diverse ways, which can lead to mutated transcripts susceptible to nonsense mediated decay, truncated unstable proteins products targeted for degradation, or reduced activity protein variants [4].

$D M D$ duplications are up to five times under-represented compared to deletions in public databases [3]. $D M D$ duplications have historically posed a technical detection challenge as exonic duplications could not be captured by PCR-based techniques, fell outside interrogated probes by multiplex ligation-dependent probe amplification (MLPA), or were under the limit of detection of chromosome microarray (CMA). Such technical difficulties are coupled with the fact that duplications can adopt different structural configurations in the genome. These include duplications in tandem direct or inverted orientations, or insertions into an entirely different chromosome, which can significantly alter the functional impact of duplications. These limitations can challenge the clinical assessment of newly identified duplications in young asymptomatic individuals based solely on genomic data; for these individuals, further molecular and protein truncation characterizations need to be performed in order to provide an accurate diagnosis.

In this report, we illustrate the molecular characterization of a $1.18 \mathrm{Mbp} D M D$ duplication spanning exons 1-7 in a 2-month-old male with mildly elevated CK levels. The duplication was found to expand up to $698 \mathrm{~Kb}$ beyond $D M D$ 's promoter regions and had a tandem configuration in direct orientation, which led to our reclassification of the variant as likely benign. Follow-up studies at 13 months of age indicated decreasing CK levels and normal development in this individual, further supporting the likely benign classification of the duplication. We hope our study prompts further characterization of $D M D$ duplications not only in young asymptomatic individuals but also in symptomatic patients, to further elucidate the impact of genomic duplications on $D M D$ transcription and dystrophin structure and improve our technical and interpretative capabilities for DMD and BMD diagnosis.

\section{Results}

\section{Case presentation}

A 2-month-old Caucasian male (proband, IV-3 in Figure 1) was referred for genetic testing due to a duplication of $D M D$ exons 1-7 identified in his mother (III-6 in Figure 1) during prenatal carrier screening by an outside laboratory; the duplication was classified as pathogenic by that laboratory. Proband was born at 39 weeks gestation by cesarean section; upon birth he presented with jaundice, mild erythema toxicum neonatorum, and neonatal hypoglycemia that resolved with feeding. His CK levels at two months of age were $324 \mathrm{U} / \mathrm{L}$. His mother is a 33-year-old P1G1 Caucasian female with a history of infertility. She presented 
with gestational hypertension without evidence of preeclampsia during pregnancy and had normal electrocardiogram results after prenatal screening follow-up for potential cardiovascular disorders conferred by the detected $D M D$ duplication. There is no family history of musculoskeletal concerns or cardiomyopathy for either of proband's parents.

\section{Genetic testing}

Diagnostic MLPA testing was performed on proband, targeting allDMD coding exons as well as its 5'UTR, 3'UTR, and DP427c regions. We identified a duplication spanning from the 5'UTR to exon 7 (g.(?_33229574)_(32827735_32717219)) (hg19). Subsequent CMA analysis of proband revealed the duplication to be 1.18Mbp in size, overlapping the 5'UTR and exons 1-7 of DMD (NM_004006) (arr[hg19] Xp21.1(32,741,375$33,926,846) \times 3$ mat) (Supplemental Figure 1). To further characterize the structural configuration of the duplication, PCR primers were designed bordering the identified duplication breakpoints, such that it could distinguish between direct tandem orientation, inverted tandem orientation, and insertion elsewhere in the genome (Supplemental Figure 2 and Supplemental Data). A long-range PCR protocol using these primers yielded a product $\sim 1.8 \mathrm{~Kb}$ in size (Supplemental Figure 3 ). Sanger sequencing of the amplified PCR product showed the duplication breakpoints to fall at Xp21.1:32,741,022 and Xp21.1:33,928,069 (hg19, Supplemental Figure 4 and Figure 2). Duplication breakpoint analysis revealed the presence of short palindromic sequences at the junction as well as microhomologies in neighboring regions (Supplemental Data).

\section{Discussion}

We molecularly characterized a novel contiguous $1.18 \mathrm{Mbp}$ duplication in $D M D$ including its 5 'UTR up to exon 7 in a two-month-old male with mildly elevated CK levels (324 U/L). The duplication was maternally inherited and initially discovered during prenatal carrier screening; the prenatal analysis reported this rearrangement to be a pathogenic exon 1-7 duplication, with the child being at a 50\% risk of developing DMD. Follow-up diagnostic analyses of proband in our laboratory included MLPA, CMA, and Sanger sequencing; we showed this duplication to be in direct tandem orientation within Xp21.1, extending further away from exon 1 and including $D M D$ 's 5'UTR as well as the DP427c alternative promoter/exon1 (Figure 2).

Sanger sequencing showed the duplication to encompass Xp21.1:32,741,022-33,928,069. There are $\sim 570 \mathrm{~Kb}$ of intervening sequence separating $D M D$ 's DP427c isoform start (neuronal and retinal isoform)[5] and the duplication end, and $\sim 68 \mathrm{~Kb}$ separating the duplication end from Dp $427 \mathrm{~m}$, which is the main isoform produced in muscle and is involved in DMD and BMD (Figure 2)[5]. While we cannot rule out splicing defects in the normal $D M D$ transcript caused by the presence of the duplicated $D M D$ exons 1-7, the intervening 570 $698 \mathrm{~Kb}$ of sequence between the duplication and the two main DMD promoters led us to hypothesize this duplication to be likely benign, as no promoter or regulatory regions were separated from the main gene body, and any potentially truncated $D M D$ product would likely undergo nonsense mediated decay (NMD). Our hypothesis was further supported by the observation that, at thirteen months of age, new measurements of CK levels in proband showed a reduction to $211 \mathrm{U} / \mathrm{L}$ (original measure was $324 \mathrm{U} / \mathrm{L}$ ). In addition, proband remains asymptomatic and shows normal development without any noticeable neuromuscular issues or motor delays. Splicing analysis remains to be performed in future experiments, as we are currently unable to complete protein truncation testing (PTT) in proband due to the requirement of a muscle biopsy which is not recommended in an asymptomatic young child [6]. Family studies are being pursued, including testing of males II-8, II-9, III-9, III-11, and IV-4 (Figure 1), to further clarify the significance of the duplication.

At the sequence level, the duplication described herein overlaps the duplication hotspot previously described for $D M D$, encompassing exons 2-20 [4]. The majority of DMD duplications have different sizes and are nonrecurrent events. These findings support non-homologous end-joining as a possible mechanism of generation. Junction analysis of the $1.18 \mathrm{Mbp}$ duplication in proband revealed the presence of microhomology, which also suggests a possible origin through microhomology-mediated mechanisms such as fork stalling and template switching (FoSTeS)[7] and microhomology-mediated break-induced replication (MMBIR)[8]. Microhomology tracts have been previously reported in the analysis of complex $D M D$ rearrangements [9], suggesting that several mechanisms can participate in DMD and BMD pathogenesis. 
We surmise the exon 1-7 duplication was given a pathogenic classification by the prenatal screening laboratory based on a previous study that had reported an exon 1-7 duplication in an individual with the Duchenne phenotype [10]. Moreover, the mild CK elevation in proband could have prompted such overdiagnosis. Currently most laboratories use a Gaussian distribution of $0-200 \mathrm{IU} / \mathrm{L}$ as the normal CK range in white individuals, with affected males having ranges >504 IU/L; however, it has been shown that CK levels in healthy populations can be skewed toward higher values, which can lead to overdiagnosis of mild abnormal values [11]. Said issue poses a dilemma to the correct diagnosis of asymptomatic infants with variants of uncertain significance (VUS) in DMD and mild CK elevations. In such instances the comprehensive characterization of the identified VUSs, particularly duplications, is paramount to the correct prediction and diagnosis of dystrophinopathies.

\section{Conclusion}

Altogether, we have illustrated the importance of molecular characterization of $D M D$ duplications in the clinical diagnosis of asymptomatic and young individuals. While duplications may escape finer characterizations due to current technical limitations, establishing their correct sizes and orientation is paramount to delivering an accurate clinical interpretation. As next generation sequencing becomes more widely available in DMD clinical testing, further molecular characterizations of duplication junctions in $D M D$ can help elucidate and predict at-risk recombination sites, and better inform our diagnostic capabilities.

\section{Materials and Methods}

Clinical DMD MLPA analysis was performed on proband to confirm the presence of the duplication, assess its size, and confirm a DMD diagnosis. MLPA experiments included probes for each of the 79DMD coding exons, 3'UTR, 5'UTR, one probe for the alternative promoter/exon $1 \mathrm{DP} 427 \mathrm{c}$, as well as control probes (MRC Holland). Capillary electrophoresis of PCR amplified ligated probe pairs was performed on ABI 3730xl (Applied Biosystems), and data analyzed with GeneMarker Software (SoftGenetics LLC, State College, PA, USA). CMA analysis was performed on proband using CytoScan HD Suite (Thermo Fisher Scientific, Waltham, MA, USA) according to manufacturer's instructions. CMA minimal and maximal probe positions for the duplication were used to design sequencing primers using Primer3Plus. Forward primer sequence: ctgtgttttgggccatttct and reverse primer sequence: tgggtttagccctaggacac produced a $1.8 \mathrm{Kbp}$ product visualized in $2 \%$ agarose gel in a UV light box. The $1.8 \mathrm{Kbp}$ product was cleaned up using Exo-SAP-IT PCR Product Cleanup Reagent (ThermoFisher Scientific, Waltham, MA, USA) and Sanger sequenced on the ABI 3730xl (Applied Biosystems, Foster City, CA, USA) according to the manufacturer's protocol. Chromatogram files were analyzed in FinchTV (Geospiza, Inc.) and FASTA files exported and mapped to hg19 using BLAT in the UCSC genome browser [12].

\section{Author Contributions}

CFF and JKGA provided clinical information; AM and CZM performed additional experiments for the molecular characterization of the DMD duplication; CZM, JEB, and LH wrote the manuscript; CZM, JEB, CFF, AM, JKGA, and LH read and approved the manuscript.

\section{Funding}

Mayo Clinic Department of Laboratory Medicine and Pathology.

\section{Conflict of Interest}

The authors declare that they have no competing interests.

\section{Ethical Approval}

Participants were consented accordingly for release of data included in this study.

\section{Acknowledgements}


We would like to thank the family for their continuous support and participation in this research report. We would also like to thank Mayo Clinic Genomics Laboratory staff for their exceptional commitment to the clinical processing and testing of these patient samples with the highest standards of quality and excellence.

\section{References}

1. Flanigan, K.M., Duchenne and Becker muscular dystrophies.Neurol Clin, 2014. 32 (3): p. 671-88, viii.

2. Gao, Q.Q. and E.M. McNally, The Dystrophin Complex: Structure, Function, and Implications for Therapy. Compr Physiol, 2015.5 (3): p. 1223-39.

3. Fokkema, I.F., et al., LOVD v.2.0: the next generation in gene variant databases. Hum Mutat, 2011. 32 (5): p. 557-63.

4. Aartsma-Rus, A., et al., Entries in the Leiden Duchenne muscular dystrophy mutation database: an overview of mutation types and paradoxical cases that confirm the reading-frame rule. Muscle Nerve, 2006. 34 (2): p. 135-44.

5. Muntoni, F., S. Torelli, and A. Ferlini, Dystrophin and mutations: one gene, several proteins, multiple phenotypes. Lancet Neurol, 2003. 2 (12): p. 731-40.

6. Aartsma-Rus, A., I.B. Ginjaar, and K. Bushby, The importance of genetic diagnosis for Duchenne muscular dystrophy. J Med Genet, 2016.53 (3): p. 145-51.

7. Lee, J.A., C.M. Carvalho, and J.R. Lupski, A DNA replication mechanism for generating nonrecurrent rearrangements associated with genomic disorders. Cell, 2007. 131 (7): p. 1235-47.

8. Hastings, P.J., G. Ira, and J.R. Lupski, A microhomology-mediated break-induced replication model for the origin of human copy number variation. PLoS Genet, 2009. 5 (1): p. e1000327.

9. Baskin, B., et al., Complex genomic rearrangements in the dystrophin gene due to replication-based mechanisms. Molecular genetics \& genomic medicine, 2014. 2 (6): p. 539-547.

10. Mah, J.K., et al., A population-based study of dystrophin mutations in Canada. Can J Neurol Sci, 2011. 38 (3): p. 465-74.

11. Moghadam-Kia, S., C.V. Oddis, and R. Aggarwal, Approach to asymptomatic creatine kinase elevation. Cleveland Clinic journal of medicine, 2016. 83 (1): p. 37-42.

12. Kuhn, R.M., D. Haussler, and W.J. Kent, The UCSC genome browser and associated tools. Brief Bioinform, 2013. 14 (2): p. 144-61.

\section{Figure legends}

Figure 1. Pedigree showing the maternal segregation of a 1.18Mbp duplication at Xp21.1 overlapping DMD. Proband is indicated with a black arrow (IV-2). There is no reported history of cardiomyopathy or dystrophinopathy in maternal and paternal families.

Figure 2. Schematic representation of $D M D$ and the duplicated segment encompassing exons 1-7, 5'UTR, Dp $427 \mathrm{~m}$, and Dp427c promoters. Notice the large intervening sequence separating the isoform promoters from the exon 7 in the duplication (dotted rectangle). 


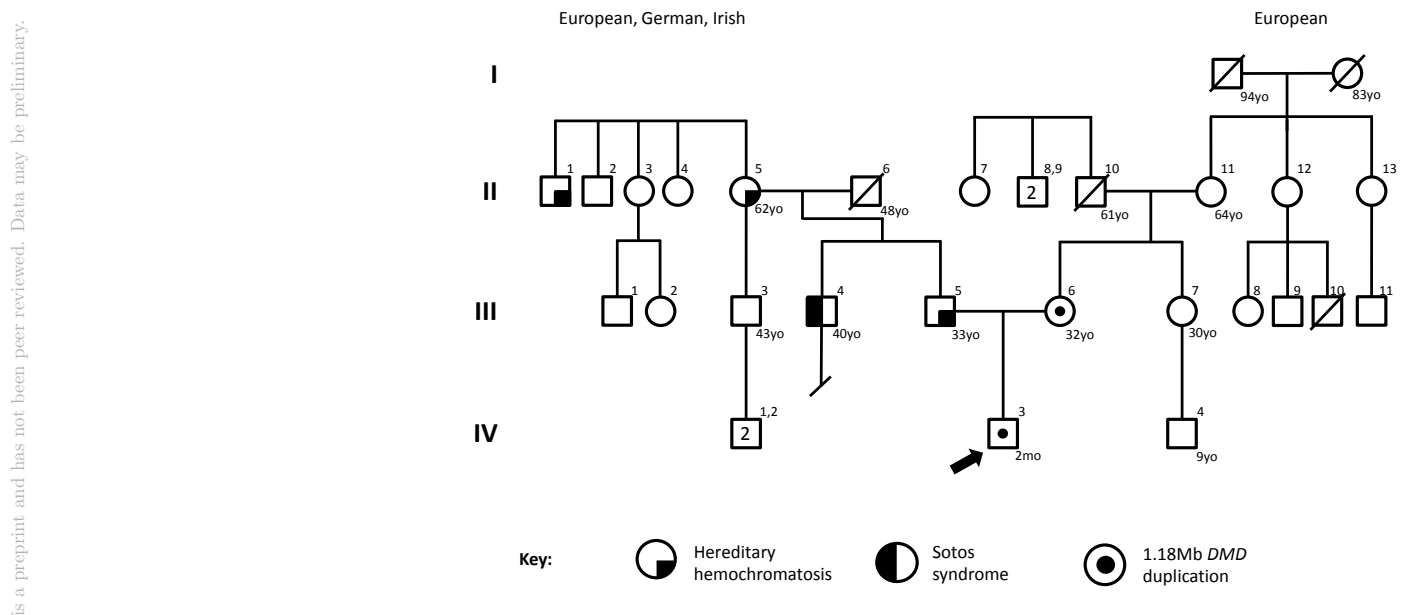

Figure 1
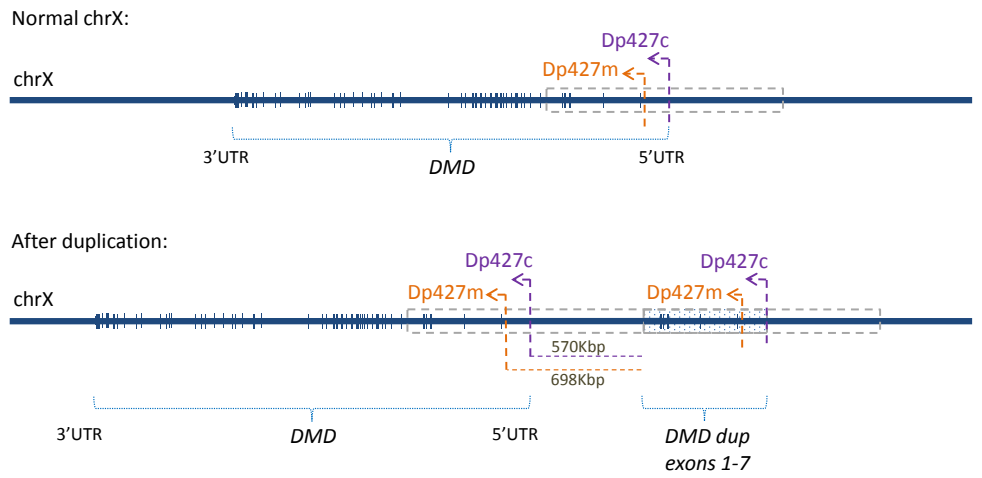

Figure 2

6 